J. Dairy Sci. 97:7307-7315

http://dx.doi.org/10.3168/jds.2013-7849

(C) American Dairy Science Association ${ }^{\circledR}, 2014$.

\title{
Stability of fatty acid composition after thermal, high pressure, and microwave processing of cow milk as affected by polyunsaturated fatty acid concentration
}

\author{
L. M. Rodríguez-Alcalá, ${ }^{*}$ L. Alonso, $†$ and J. Fontecha* \\ *Department of Bioactivity and Food Analysis, Group of Lipids, Instituto de Investigación en Ciencias de la Alimentación (CIAL CSIC-UAM), \\ Nicolás Cabrera, 9, Universidad Autónoma de Madrid, 28049, Madrid, Spain \\ †Instituto de Productos Lácteos de Asturias (IPLA CSIC), Carretera de Infiesto s/n, 33300 Villaviciosa, Principado de Asturias, Spain
}

\begin{abstract}
Interest has been increasing to enhance the contents of healthy polyunsaturated fatty acid (PUFA) in milk. However, trans fatty acids and conjugated linoleic acid (CLA) can be altered after thermal processing and high pressures disrupt the milk fat globule membrane, exposing the lipid core and helping its oxidation. The objective of the present research was to study whether processing can alter the fatty acid composition of milk and if these changes are affected by PUFA concentration as previous studies suggest. Two cow milk batches $(500 \mathrm{~L}$ each), one naturally enriched in PUFA, were processed to obtain pasteurized; high temperature, short time; UHT; high pressure; and microwave pasteurized samples. The detailed fatty acid composition was analyzed with special attention to trans fatty acids and CLA isomers. Results showed that after high temperature, short time processing, total CLA content increased in both milk batches, whereas sterilization resulted in a sigmatropic rearrangement of $\mathrm{C} 18: 2$ cis-9,trans-11 to C18:2 trans-9,trans-11. The extent of these effects was greater in milks naturally enriched in PUFA.
\end{abstract}

Key words: milk processing, conjugated linoleic acid isomers, trans fatty acids, unsaturated fatty acids

\section{INTRODUCTION}

Because of the relatively high amount of SFA and trans fatty acids (TFA) in dairy fat and the role of those compounds in the risk of chronic and other metabolic diseases, food industries are committed to decrease SFA, cholesterol, and TFA contents as much as is possible (Nishida et al., 2004; Haug et al., 2007). The high susceptibility of milk fat profile modulation to diet composition has led to the carrying out of an extensive number of research works supplementing

Received December 18, 2013.

Accepted August 5, 2014.

${ }^{1}$ Corresponding author: j.fontecha@csic.es forage/ensilage diets with high content PUFA oils or oilseeds and resulting in dairy products naturally enriched in polyunsaturated bioactive lipids (from 3.6 to $4.8 \mathrm{~g}$ of PUFA $/ 100 \mathrm{~g}$ of fat with control diets to $9 \mathrm{~g}$ of PUFA/100 g of fat with supplemented feeding) and lower SFA concentrations (from 65 to $68 \mathrm{~g}$ of PUFA/100 $\mathrm{g}$ of fat with control diets to $55-47 \mathrm{~g}$ of PUFA/100 g of fat with supplemented feeding; Jones et al., 2005; Lynch et al., 2005; Jenkins and McGuire, 2006).

Furthermore, PUFA have been demonstrated to exert important beneficial activities in human health: linoleic acid (C18:2 cis (c)-9c12) is a precursor of arachidonic acid, involved in the synthesis of molecules associated to inflammatory processes (Calder, 2006), and linolenic acid (C18:3 c9c12c15) is a precursor of eicosapentaenoic, docosapentaenoic, and docosahexaenoic acids (Williams, 2000). The rate of these $2 \mathrm{FA}(\mathrm{n}-6 / \mathrm{n}-3)$ in human erythrocytes is related to cardiovascular risk (Harris, 2008). Even more, CLA has been extensively associated with anticancer (Hagen et al., 2013) and body weight properties (Rodríguez-Alcalá et al., 2013b).

In milk, oxidation of lipids produces strong off-flavors and deterioration of the nutritional and health quality, affecting both the shelf-life and acceptability of the product by consumers (Rafalowski et al., 2014). These reactions are affected by the degree of unsaturation of the FA, and PUFA are highly prone to oxidation, mainly when radical starters are present. Because bioactivity of these compounds is associated with a dose [e.g., $3 \mathrm{~g}$ of CLA/d according to Ip et al. (1996)], any possible degradation would result in a daily intake below the amount needed to obtain the beneficial effect. Thus, in dual-homogenized $(20,000 \mathrm{kPa})$ skim milk fortified with CLA oil, total trans trans CLA isomers concentration increased, whereas total double-bond cis cis decreased after industrial atomization processing (RodríguezAlcalá and Fontecha, 2007). Studies focused on the effects of pasteurization (homogenization at 13,789 $\mathrm{kPa}$; $77.2^{\circ} \mathrm{C}, 12 \mathrm{~s}$ ) reported that rumenic acid but not $\mathrm{C} 18: 2$ trans (t)-10,cis-12 CLA decreased within $24 \mathrm{~h}$ after treatment and refrigerated storage at $5^{\circ} \mathrm{C}$ (Campbell et 
al., 2003). Herzallah et al. (2005b) found that low pasteurization $\left(63^{\circ} \mathrm{C}, 10 \mathrm{~min}\right)$ and microwaving $\left(96.8^{\circ} \mathrm{C}, 5\right.$ min) processing of milk caused losses of TFA whereas HTST after $3 \mathrm{~d}$ at $5^{\circ} \mathrm{C}\left(85^{\circ} \mathrm{C}, 16 \mathrm{~s}\right)$, UHT after $5 \mathrm{~d}$ $\left(140^{\circ} \mathrm{C}, 4 \mathrm{~s}\right)$, and microwave processing decreased the total concentration of CLA.

As a solution to this, new emerging technologies such as high pressure processing and homogenization (50-1,000 MPa) are being studied to evaluate their potential as alternative or complementary process to thermal pasteurization as $400 \mathrm{MPa}, 15$ min are conditions able to kill most bacteria in milk (Urala and Lahteenmaki, 2007; Chawla et al., 2011). However, it has not been found modifications of the lipid fraction when human (0-600 MPa) or cow, goat, and sheep milk (0-350 MPa) were processed by high pressure or high pressure homogenization (Rodríguez-Alcalá et al., 2009; Moltó-Puigmartí et al., 2011). It is known that those treatments can result in partial whey protein denaturation, casein micelle dissociation, and disruption of the milk fat globule membrane (Pereda et al., 2007; Zamora et al., 2007). There is also evidence that 200 $\mathrm{MPa}, 30 \mathrm{~min}$ and inlet temperature of $60^{\circ} \mathrm{C}$ increased the native lipase activity when compared with raw milk (Datta et al., 2005).

The aim of the present work is to study the possible effects of conventional heating (pasteurization, UHT, and sterilization), high pressure, and microwave processing on the fatty acid composition of naturally PUFA-enriched milk with special attention to CLA isomers and TFA.

\section{MATERIALS AND METHODS}

\section{Chemicals}

Hexane, methanol, and chloroform were purchased from LabScan (Dublin, Ireland); potassium hydroxide, sodium sulfate- 1 hydrate, and $20 \%$ aqueous solution of $\mathrm{AgNO}_{3}$ from Panreac (Barcelona, Spain); CLA standards from Nu-Chek Prep (Elysian, MN); stearic FAME (C18:0), elaidic FAME (C18:1 t9), rumenic acid (C18:2 c9,t11; RA), 1,2,3-tritridecanoylglycerol, and sodium azide from Sigma (St. Louis, MO); and high CLA concentration oil (Tonalin) was obtained from Cognis (Illertissen, Germany). All reagents were GC or HPLC grade. Reference milk fat butter BCR-164 (EU Commissions; Brussels, Belgium) was purchased from Fedelco Inc. (Madrid, Spain).

\section{Samples}

Two raw milk batches of $500 \mathrm{~L}$ labeled as B1 (batch 1) and B2 (batch 2), collected from different cows herds
(Holstein breed) from the Castilla-La Mancha region (Spain) were kindly donated by a Spanish dairy company (Leches Pascual, Aranda de Duero, Spain). Animals producing the B2 samples were fed with linseed. Milk was processed to obtain pasteurized $\left(72^{\circ} \mathrm{C}, 30 \mathrm{~s}\right)$, $\operatorname{HTST}\left(85^{\circ} \mathrm{C}, 30 \mathrm{~s}\right)$, and $\mathrm{UHT}\left(\mathbf{U H T} 1,135^{\circ} \mathrm{C}, 30 \mathrm{~s}\right.$, and UHT2, $150^{\circ} \mathrm{C}, 5 \mathrm{~min}$ ) samples using an aseptic sterilizer (Rossi \& Catelli, Parma, Italy) as follows: $30 \mathrm{~L}$ of raw milk per assay were preheated to $65^{\circ} \mathrm{C}$ and then homogenized at $18 \mathrm{MPa}$. The temperature was raised to pasteurization conditions and then cooled to an output value of $20^{\circ} \mathrm{C}$. In UHT processing, the preheating step was carried out after homogenization $\left(85^{\circ} \mathrm{C}\right)$. Sterilization (STR, $121^{\circ} \mathrm{C}, 15 \mathrm{~min}$ ) was carried out using $1 \mathrm{~L}$ of raw milk from each batch in a laboratory autoclave (Selecta Autotester E DRY-PV, Barcelona, Spain). As control samples $4 \mathrm{~L}$ of raw milk were collected from the 2 batches. For high pressure processing (400 MPa, $25^{\circ} \mathrm{C}, 15 \mathrm{~min}$ ), a laboratory-scale high pressure machine (ACB, GEC, Alsthom, Nantes, France) was used with $200 \mathrm{~mL}$ of raw milk fitted in pressure-resistant packages. Microwaving (650 W, $1.30 \mathrm{~min})$ was performed using $100 \mathrm{~mL}$ of raw milk in a domestic apparatus (Moulinex AET1, Ecully Cedex, France). All samples were placed into amber-glass bottles and sodium azide $(0.06 \mathrm{~g} / \mathrm{mL})$ added to avoid microbial growth. All samples were placed in refrigeration after processing. Assays were performed in duplicate.

\section{Basic Parameter Analysis}

Fat content, protein, lactose, and dried extract were measured using a Milkoscan (Foss, Hillerød, Denmark).

\section{Lipid Extraction and FA Derivatization}

Milk fat extraction was carried out according to standard methods (ISO-IDF, 2001). The fat residue was collected into amber vials and stored at $-20^{\circ} \mathrm{C}$ until analysis. Fatty acid methyl esters were prepared by base-catalyzed methanolysis (2 $N \mathrm{KOH}$ in methanol) according to ISO-IDF (2002). As internal standard, 1,2,3-tritridecanoylglycerol, was added to samples (200 $\mu \mathrm{L}, 1.24 \mathrm{mg} / \mathrm{mL}$ ). All analyses of samples were carried out in duplicate.

\section{Fractioning by $\mathrm{AgNO}_{3}$-Thin Layer Chromatography of FAME}

Fatty acid methyl esters were fractionated according to the number and geometry of double bounds by thin layer chromatography (TLC) according to Alonso et al. (1999). The TLC glass plates $(20 \times 20 \mathrm{~cm})$ with silica gel (0.25 mm; Merck, Darmstadt, Germany) were 
incubated with $20 \%$ aqueous solution of $\mathrm{AgNO}_{3}$ for 16 $\mathrm{h}$, were partially air-dried, and activated at $120^{\circ} \mathrm{C}$ for 30 min. A $100-\mu \mathrm{L}$ solution of FAME $(100 \mathrm{mg} / \mathrm{mL})$ was applied to the activated TLC glass plate in a narrow band. The plate was developed twice in a saturated chamber in hexane and diethyl ether $(9: 1, \mathrm{vol} / \mathrm{vol})$ with $15 \mathrm{~cm}$ migration. At the end of chromatographic runs, the plates were air-dried and sprayed with a $0.20 \%$ ethanol solution of $2^{\prime}, 7^{\prime}$-dichlorofluorescein, and the bands were visualized under UV light. The bands corresponding to saturated and trans monoenoic FAME, which were previously identified by a mixture of stearic FAME (C18) and elaidic FAME (C18:1 t9) running in $\mathrm{AgNO}_{3}$-TLC, were scraped into a flask. The FAME were extracted with $80 \mathrm{~mL}$ of diethyl ether in 4 extractions, and the solvent was evaporated in a rotary evaporator and stream of nitrogen. The residue was dissolved in $200 \mu \mathrm{L}$ of hexane and used for GC analysis.

To calculate the total content of trans C18:1 isomers, the ratio of $\mathrm{C} 18$ to total trans $\mathrm{C} 18: 1$ was determined in the saturated plus trans monoenoic $\mathrm{AgNO}_{3}$-TLC fraction and was related to the C18:0 content of total FAME. To calculate the total content of trans C16:1 isomers, the ratio of total $\mathrm{C} 16: 1$ t to $\mathrm{C} 18: 1$ t10 plus C18:1 t11 was determined in the trans monoenoic $\mathrm{AgNO}_{3}$-TLC fraction and related to the ratio of $\mathrm{C} 18$ to $\mathrm{C} 18: 1$ t10 plus $\mathrm{C} 18: 1 \mathrm{t} 11$ in the saturated plus trans monoenoic $\mathrm{AgNO}_{3}$-TLC fraction and to the $\mathrm{C} 18$ content of total FAME. All analyses were performed at least in triplicate.

\section{GC-Flame-Ionization Detection Analyses of Total FA and TFA}

Fatty acid methyl esters were analyzed on a Clarus 500 gas chromatograph (Perkin Elmer, Beaconsfield, UK) with a VF23ms, fused-silica capillary column (30 $\mathrm{m} \times 0.25 \mathrm{~mm}$ i.d. $\times 0.25 \mu \mathrm{m}$ film thickness, Varian, Middelburg, the Netherlands). Quick chromatography conditions were as previously described by RodríguezAlcalá et al. (2013a).

Trans FA, minor cis octadecenoic (C18:1 c), and octadecadienoic FA isomers were analyzed in an Autosystem chromatograph (Perkin-Elmer) with a flame-ionization detector. Fatty acids methyl esters were separated using a CP-Sil 88 fused-silica capillary column $(100 \mathrm{~m} \times 0.25 \mathrm{~mm}$ i.d. $\times 0.2 \mu \mathrm{m}$ film thickness, Chrompack, Middelburg, the Netherlands). The column was held at $100^{\circ} \mathrm{C}$ for 1 min after injection, increased at $7^{\circ} \mathrm{C} / \mathrm{min}$ to $170^{\circ} \mathrm{C}$, held there for $55 \mathrm{~min}$, increased at $10^{\circ} \mathrm{C} / \mathrm{min}$ to $230^{\circ} \mathrm{C}$ and held there for 33 min. Helium was the carrier gas, with a column inlet pressure set at $214 \mathrm{kPa}$ and a split ratio of 1: 20 . The injection volume was $0.5 \mu \mathrm{L}$.
Response factors were calculated as obtained from a certified reference butter fat (BCR-164, EU Commissions, Brussels, Belgium, purchased from Fedelco Inc.) and used for sample peak area correction. All analyses were performed at least in triplicate.

\section{Quantification of Total CLA Content by UV Spectroscopy}

For total CLA quantification, samples were weighed to obtain a concentration of $0.2 \mathrm{mg} / \mathrm{mL}$ in hexane, placed into 2-mL quartz cuvettes, and absorbance was recorded with a scan program $(190-350 \mathrm{~nm})$ and data expressed as absorbance at $233 \mathrm{~nm}$, in a Lambda650 spectrophotometer (Perkin-Elmer) as described by Rodríguez-Alcalá et al. (2011). Concentration was calculated by means of pure CLA FAME standard calibration curve.

\section{Silver-Ion HPLC $\left(\mathrm{Ag}^{+}-\mathrm{HPLC}\right)$}

Separation of CLA as FAME was performed using HPLC (Shimadzu Vp Series, Duisburg, F.R. Germany) equipped with UV detector at $233 \mathrm{~nm}$ and a ChromSpher 5 Lipid analytical column $(4.6 \mathrm{~mm}$ i.d. $\times 250$ $\mathrm{mm}$ stainless steel, $5 \mu \mathrm{m}$ particle size; Varian). The mobile phase was $0.1 \%$ acetonitrile in hexane, operated isocratically at a flow rate of $1.0 \mathrm{~mL} / \mathrm{min}$. The injection volume was $10 \mu \mathrm{L}$. For identification, a standard mixture of pure CLA FAME isomers was used.

\section{Statistical Analysis}

In the first instance, an exploratory analysis of data was performed to test normal distribution and homogeneity of variance. Then, a full factorial data analysis (one-way ANOVA and GLM) was conducted with the aid of the SPSS Statistics software v21.0 for Windows (IBM, Armonk, NY). Level of significance was fixed at $P<0.05$ and $0.05<P<0.1$ was considered a trend.

\section{RESULTS AND DISCUSSION}

\section{FA Composition of the Assayed Raw Cow Milk}

In the assayed samples, values for basic composition parameters were as follows: fat content $(3.53 \mathrm{~g} / 100 \mathrm{~mL}$ \pm 0.21 in $\mathrm{B} 1$ and $2.74 \mathrm{~g} / 100 \mathrm{~mL} \pm 0.07$ in samples B2), protein $(3.15 \mathrm{~g} / 100 \mathrm{~mL} \pm 0.01 ; 3.13 \mathrm{~g} / 100 \mathrm{~mL} \pm 0.05)$, lactose $(4.71 \mathrm{~g} / 100 \mathrm{~mL} \pm 0.07 ; 5.09 \mathrm{~g} / 100 \mathrm{~mL} \pm 0.08)$ and dried extract $(11.39 \mathrm{~g} / 100 \mathrm{~mL} \pm 0.23 ; 10.96 \mathrm{~g} / 100$ $\mathrm{mL} \pm 0.29)$.

The FA composition of the assayed samples is shown in Tables 1 and 2. When compared, the total amount 
of SFA in B2 raw milk $(58.64 \%)$ was lower $(P<0.05)$ than in B1 (66.95\%) as result of the differences in the concentration of lauric (C12), myristic (14), palmitic (C16), and stearic $(\mathrm{C} 18)$ acids $(P<0.05)$. Previous studies reported that addition of linseed (rich in linolenic acid, C18:3) to the diets of cows led to lower contents of medium-chain fatty acids $(\mathrm{C} 10-14)$ and increased the concentrations of stearic, linoleic (C18:2 c9c12), and oleic acids in milk (Lawless et al., 1998; Rego et al., 2005). These effects are related to changes in the rumen $\mathrm{pH}$ resulting in decreased acetate production (McNamee et al., 2002). This compound is directly involved in de novo synthesis of fatty acids $(\mathrm{C} 4-\mathrm{C} 14)$ in the mammary gland.

Monounsaturated fatty acids concentration was $34.85 \%$ in the $\mathrm{B} 2$ raw milk and $28.63 \%$ for B1. Thus, in this fraction, oleic acid (21.97\% in B2; $20.55 \%$ in B1),
C18:1 c (1.53\% in B1 vs. $2.46 \%$ in B2), C18:1 t (3.57\% in $\mathrm{B} 1$ vs. $7.23 \%$ in $\mathrm{B} 2)$, and $\mathrm{C} 16: 1 \mathrm{c} 9(1.84 \%$ in $\mathrm{B} 1 ; 1.65 \%$ in B2) were the main FA. According to the results of the present research, the cis and trans MUFA moieties (Tables 3 and 4) were characterized by the concentrations of $\mathrm{C} 18: 1 \mathrm{t} 10$ (0.43\% in $\mathrm{B} 1 ; 1.71 \%$ in $\mathrm{B} 2), \mathrm{C} 18: 1$ t11 (0.87\% in B1 vs. $1.82 \%$ in $\mathrm{B} 2$; trans vaccenic acid), $\mathrm{C} 18: 1 \mathrm{c} 11$ (0.71\% in B1 vs. $0.81 \%$ in B2), and $\mathrm{C} 18: 1 \mathrm{c} 12$ $(0.37 \%$ in $\mathrm{B} 1$ vs. $0.92 \% \mathrm{~B} 2 ; P<0.05)$. It has been described elsewhere that the alteration of the fermentative environment by the supplementation of the cows' diets with PUFA sources affects the metabolic pathways of the rumen biohydrogenation, increasing the concentration of the trans MUFA, mainly C18:1 t10 (Bauman et al., 2006; Elgersma et al., 2006; Li et al., 2007).

As with SFA and MUFA, similar significant differences among batches were observed for PUFA $(4.42 \%$

Table 1. Fatty acid composition ( $\mathrm{g} / 100 \mathrm{~g}$ of $\mathrm{FA}$; means $\pm \mathrm{SD})$ in $\mathrm{B} 1$ milk samples ${ }^{1}$

\begin{tabular}{|c|c|c|c|c|c|c|c|c|}
\hline Fatty acid ${ }^{2}$ & Raw & Past. & HTST & UHT1 & UHT2 & STR & $\mathrm{HP}$ & Micro. \\
\hline $\mathrm{C} 4$ & $3.36 \pm 0.36$ & $3.15 \pm 0.28$ & $3.09 \pm 0.55$ & $3.03 \pm 0.69$ & $2.85 \pm 0.69$ & $3.20 \pm 0.32$ & $3.13 \pm 0.14$ & $3.32 \pm 0.47$ \\
\hline C6 & $2.31 \pm 0.46$ & $1.99 \pm 0.17$ & $2.19 \pm 0.41$ & $2.10 \pm 0.28$ & $2.12 \pm 0.36$ & $2.11 \pm 0.26$ & $2.11 \pm 0.37$ & $2.41 \pm 0.83$ \\
\hline $\mathrm{C} 8$ & $1.26 \pm 0.22$ & $1.39 \pm 0.50$ & $1.24 \pm 0.10$ & $1.25 \pm 0.10$ & $1.31 \pm 0.13$ & $1.31 \pm 0.23$ & $1.20 \pm 0.22$ & $1.23 \pm 0.12$ \\
\hline $\mathrm{C} 10$ & $2.80 \pm 0.37$ & $2.88 \pm 0.81$ & $2.76 \pm 0.14$ & $2.80 \pm 0.17$ & $2.99 \pm 0.42$ & $2.86 \pm 0.43$ & $2.65 \pm 0.26$ & $2.74 \pm 0.15$ \\
\hline C10:1 & $0.31 \pm 0.07$ & $0.33 \pm 0.10$ & $0.30 \pm 0.05$ & $0.31 \pm 0.05$ & $0.33 \pm 0.08$ & $0.31 \pm 0.07$ & $0.28 \pm 0.05$ & $0.30 \pm 0.05$ \\
\hline $\mathrm{C} 12$ & $3.05 \pm 0.10$ & $3.27 \pm 0.40$ & $3.11 \pm 0.16$ & $3.11 \pm 0.13$ & $3.28 \pm 0.43$ & $3.12 \pm 0.32$ & $3.04 \pm 0.18$ & $3.09 \pm 0.15$ \\
\hline C14 & $9.89 \pm 0.36$ & $10.30 \pm 0.28$ & $10.28 \pm 0.26$ & $10.31 \pm 0.20$ & $10.43 \pm 0.54$ & $10.04 \pm 0.45$ & $10.05 \pm 0.28$ & $10.19 \pm 0.29$ \\
\hline $\mathrm{C} 15 i$ & $0.20 \pm 0.01$ & $0.21 \pm 0.01$ & $0.21 \pm 0.01$ & $0.21 \pm 0.01$ & $0.21 \pm 0.01$ & $0.20 \pm 0.02$ & $0.20 \pm 0.01$ & $0.21 \pm 0.01$ \\
\hline C14:1 & $0.45 \pm 0.03$ & $0.47 \pm 0.02$ & $0.46 \pm 0.02$ & $0.47 \pm 0.03$ & $0.47 \pm 0.02$ & $0.40 \pm 0.16$ & $0.45 \pm 0.03$ & $0.46 \pm 0.02$ \\
\hline $\mathrm{C} 15 a i$ & $0.84 \pm 0.03$ & $0.89 \pm 0.03$ & $0.87 \pm 0.02$ & $0.87 \pm 0.02$ & $0.88 \pm 0.04$ & $0.85 \pm 0.05$ & $0.85 \pm 0.03$ & $0.86 \pm 0.02$ \\
\hline C15 & $1.07 \pm 0.08$ & $1.11 \pm 0.03$ & $1.11 \pm 0.01$ & $1.10 \pm 0.02$ & $1.12 \pm 0.02$ & $1.08 \pm 0.05$ & $1.10 \pm 0.04$ & $1.10 \pm 0.02$ \\
\hline $\mathrm{C} 16 i$ & $0.20 \pm 0.01$ & $0.21 \pm 0.01$ & $0.21 \pm 0.01$ & $0.21 \pm 0.01$ & $0.19 \pm 0.07$ & $0.20 \pm 0.01$ & $0.21 \pm 0.01$ & $0.21 \pm 0.01$ \\
\hline $\mathrm{C} 16$ & $31.97 \pm 1.33$ & $31.83 \pm 2.08$ & $32.1 \pm 2.07$ & $32.45 \pm 1.64$ & $33.00 \pm 0.36$ & $31.9 \pm 1.92$ & $32.06 \pm 1.83$ & $32.02 \pm 1.63$ \\
\hline $\mathrm{C} 17 i$ & $0.20 \pm 0.12$ & $0.27 \pm 0.06$ & $0.27 \pm 0.07$ & $0.29 \pm 0.07$ & $0.28 \pm 0.07$ & $0.26 \pm 0.05$ & $0.25 \pm 0.05$ & $0.24 \pm 0.10$ \\
\hline C16:1 c9 & $1.84 \pm 0.04$ & $1.86 \pm 0.10$ & $1.87 \pm 0.12$ & $1.90 \pm 0.10$ & $1.93 \pm 0.03$ & $1.81 \pm 0.15$ & $1.82 \pm 0.15$ & $1.86 \pm 0.10$ \\
\hline $\mathrm{C} 17$ & $0.53 \pm 0.02$ & $0.52 \pm 0.04$ & $0.53 \pm 0.04$ & $0.53 \pm 0.03$ & $0.55 \pm 0.01$ & $0.53 \pm 0.03$ & $0.53 \pm 0.04$ & $0.53 \pm 0.03$ \\
\hline $\mathrm{C} 17: 1$ & $0.24 \pm 0.05$ & $0.25 \pm 0.04$ & $0.25 \pm 0.04$ & $0.25 \pm 0.04$ & $0.25 \pm 0.04$ & $0.25 \pm 0.05$ & $0.26 \pm 0.05$ & $0.24 \pm 0.05$ \\
\hline C18 & $9.10 \pm 0.41$ & $8.99 \pm 0.82$ & $9.15 \pm 0.71$ & $9.32 \pm 0.52$ & $9.56 \pm 0.10$ & $9.13 \pm 0.77$ & $9.18 \pm 0.73$ & $9.17 \pm 0.60$ \\
\hline $\mathrm{C} 18: 1 \mathrm{t}^{3}$ & $3.57 \pm 0.11$ & $3.43 \pm 0.35$ & $3.38 \pm 0.33$ & $3.40 \pm 0.24$ & $3.16 \pm 0.08$ & $3.48 \pm 0.20$ & $3.60 \pm 0.15$ & $3.41 \pm 0.26$ \\
\hline C18:1 c9 & $20.55 \pm 1.02$ & $20.68 \pm 1.45$ & $20.49 \pm 1.39$ & $20.21 \pm 1.25$ & $19.52 \pm 0.45$ & $20.71 \pm 1.24$ & $20.76 \pm 1.54$ & $20.49 \pm 1.69$ \\
\hline $\mathrm{C} 18: 1 \mathrm{c}^{4}$ & $1.53 \pm 0.09$ & $1.43 \pm 0.19$ & $1.44 \pm 0.11$ & $1.40 \pm 0.11$ & $1.24 \pm 0.06$ & $1.55 \pm 0.10$ & $1.59 \pm 0.13$ & $1.40 \pm 0.14$ \\
\hline $\mathrm{C} 18: 2$ c9c12 & $2.63 \pm 0.10$ & $2.46 \pm 0.24$ & $2.49 \pm 0.16$ & $2.43 \pm 0.17$ & $2.36 \pm 0.28$ & $2.54 \pm 0.18$ & $2.53 \pm 0.16$ & $2.47 \pm 0.18$ \\
\hline C18:2 other ${ }^{5}$ & $0.65 \pm 0.03$ & $0.54 \pm 0.07$ & $0.59 \pm 0.05$ & $0.57 \pm 0.05$ & $0.51 \pm 0.06$ & $0.61 \pm 0.06$ & $0.59 \pm 0.06$ & $0.56 \pm 0.05$ \\
\hline $\mathrm{C} 20$ & $0.13 \pm 0.03$ & $0.12 \pm 0.02$ & $0.12 \pm 0.03$ & $0.11 \pm 0.03$ & $0.11 \pm 0.02$ & $0.13 \pm 0.03$ & $0.12 \pm 0.04$ & $0.12 \pm 0.02$ \\
\hline C20:1 & $0.14 \pm 0.02$ & $0.16 \pm 0.02$ & $0.16 \pm 0.01$ & $0.16 \pm 0.02$ & $0.15 \pm 0.01$ & $0.17 \pm 0.02$ & $0.16 \pm 0.02$ & $0.10 \pm 0.06$ \\
\hline $\mathrm{C} 18: 3$ & $0.31 \pm 0.01$ & $0.33 \pm 0.04$ & $0.33 \pm 0.03$ & $0.33 \pm 0.03$ & $0.30 \pm 0.02$ & $0.32 \pm 0.02$ & $0.33 \pm 0.03$ & $0.33 \pm 0.03$ \\
\hline C18:2 c9t11 & $0.39 \pm 0.02$ & $0.43 \pm 0.04$ & $0.43 \pm 0.03$ & $0.43 \pm 0.02$ & $0.39 \pm 0.04$ & $0.41 \pm 0.03$ & $0.41 \pm 0.04$ & $0.43 \pm 0.03$ \\
\hline CLA other & $0.14 \pm 0.02$ & $0.13 \pm 0.04$ & $0.14 \pm 0.03$ & $0.13 \pm 0.04$ & $0.13 \pm 0.04$ & $0.13 \pm 0.04$ & $0.14 \pm 0.03$ & $0.14 \pm 0.04$ \\
\hline $\mathrm{C} 22$ & $0.04 \pm 0.02$ & $0.05 \pm 0.01$ & $0.05 \pm 0.01$ & $0.05 \pm 0.01$ & $0.05 \pm 0.01$ & $0.06 \pm 0.03$ & $0.05 \pm 0.01$ & $0.05 \pm 0.02$ \\
\hline $\mathrm{C} 20: 3$ & $0.12 \pm 0.01$ & $0.14 \pm 0.02$ & $0.14 \pm 0.02$ & $0.14 \pm 0.01$ & $0.13 \pm 0.01$ & $0.12 \pm 0.04$ & $0.13 \pm 0.01$ & $0.14 \pm 0.01$ \\
\hline $\mathrm{C} 20: 4 \mathrm{ARA}^{6}$ & $0.18 \pm 0.01$ & $0.20 \pm 0.03$ & $0.21 \pm 0.03$ & $0.21 \pm 0.03$ & $0.19 \pm 0.02$ & $0.19 \pm 0.02$ & $0.20 \pm 0.03$ & $0.20 \pm 0.03$ \\
\hline Total SFA & $66.95 \pm 1.18$ & $67.17 \pm 1.77$ & $67.31 \pm 1.70$ & $67.66 \pm 1.27$ & $68.94 \pm 0.75$ & $67.01 \pm 1.49$ & $66.74 \pm 1.74$ & $67.48 \pm 1.76$ \\
\hline Total MUFA & $28.63 \pm 1.12$ & $28.6 \pm 1.63$ & $28.36 \pm 1.57$ & $28.11 \pm 1.14$ & $27.05 \pm 0.42$ & $28.68 \pm 1.45$ & $28.93 \pm 1.70$ & $28.25 \pm 1.61$ \\
\hline Total PUFA & $4.42 \pm 0.07$ & $4.23 \pm 0.39$ & $4.33 \pm 0.22$ & $4.23 \pm 0.21$ & $4.01 \pm 0.39$ & $4.32 \pm 0.18$ & $4.33 \pm 0.17$ & $4.27 \pm 0.24$ \\
\hline
\end{tabular}

${ }^{1}$ Past. = low temperature pasteurization $\left(72^{\circ} \mathrm{C}, 30 \mathrm{~s}\right) ; \mathrm{HTST}=\mathrm{HTST}$ pasteurization $\left(85^{\circ} \mathrm{C}, 30 \mathrm{~s}\right) ; \mathrm{UHT} 1=\mathrm{UHT}$ processing $\left(135^{\circ} \mathrm{C}, 30 \mathrm{~s}\right) ; \mathrm{UHT} 2$ $=$ non-commercial UHT processing $\left(150^{\circ} \mathrm{C}, 5 \mathrm{~min}\right) ; \mathrm{STR}=$ sterilization $\left(121^{\circ} \mathrm{C}, 15 \mathrm{~min}\right) ; \mathrm{HP}=$ high pressure processing $\left(400 \mathrm{MPa}, 25^{\circ} \mathrm{C}, 15\right.$ $\min )$; Micro. $=$ microwave pasteurization $(650 \mathrm{~W}, 1.30 \mathrm{~min})$.

${ }^{2} i=$ iso; ai $=$ anteiso; $\mathrm{c}=$ cis; $\mathrm{t}=$ trans.

${ }^{3} \mathrm{C} 18: 1 \mathrm{t}$ (t4, t5, t6-t8, t9, t10, t11, t12, t13-t14, t15, t16).

${ }^{4} \mathrm{C} 18: 1 \mathrm{c}(\mathrm{c} 11, \mathrm{c} 12, \mathrm{c} 13, \mathrm{c} 14, \mathrm{c} 15)$.

${ }^{5} \mathrm{C} 18: 2$ other $(\mathrm{C} 18: 2 \mathrm{t}, \mathrm{t}$ NMID, t9,t12, t9,t13, t9,t12), where NMID = non-methylene-interrupted dienoic.

${ }^{6} \mathrm{ARA}=$ arachidonic acid. 
Table 2. Fatty acid composition (g/100 g of FA; means \pm SD) in B2 milk samples ${ }^{1}$

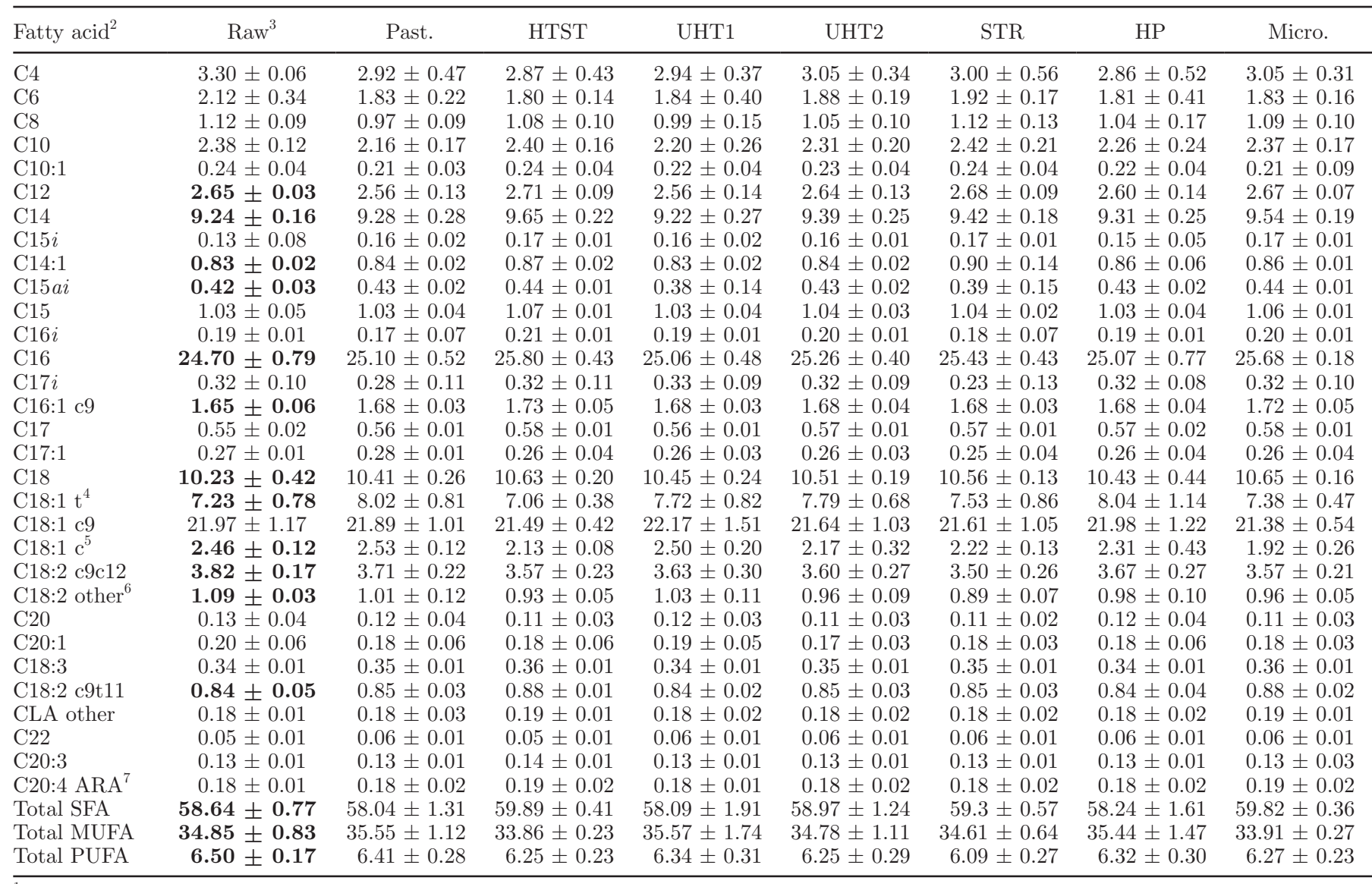

${ }^{1}$ Past. = low temperature pasteurization $\left(72^{\circ} \mathrm{C}, 30 \mathrm{~s}\right) ; \mathrm{HTST}=\mathrm{HTST}$ pasteurization $\left(85^{\circ} \mathrm{C}, 30 \mathrm{~s}\right) ; \mathrm{UHT} 1=\mathrm{UHT}$ processing $\left(135^{\circ} \mathrm{C}, 30 \mathrm{~s}\right) ; \mathrm{UHT} 2$ $=$ non-commercial $\mathrm{UHT}$ processing $\left(150^{\circ} \mathrm{C}, 5 \mathrm{~min}\right) ; \mathrm{STR}=$ sterilization $\left(121^{\circ} \mathrm{C}, 15 \mathrm{~min}\right) ; \mathrm{HP}=$ high pressure processing $\left(400 \mathrm{MPa}, 25^{\circ} \mathrm{C}, 15\right.$ $\min )$; Micro. $=$ microwave pasteurization $(650 \mathrm{~W}, 1.30 \mathrm{~min})$.

${ }^{2} i=i s o ;$ ai $=$ anteiso $; \mathrm{c}=$ cis; $\mathrm{t}=$ trans.

${ }^{3}$ Bold numbers in raw milk sample column indicate significant differences among batches $(P<0.05)$.

${ }^{4} \mathrm{C} 18: 1 \mathrm{t} \mathrm{(t4,} \mathrm{t5,} \mathrm{t6-t8,} \mathrm{t9,} \mathrm{t10,} \mathrm{t11,} \mathrm{t12,} \mathrm{t13-t14,} \mathrm{t15,} \mathrm{t16).}$

${ }^{5} \mathrm{C} 18: 1 \mathrm{c}(\mathrm{c} 11, \mathrm{c} 12, \mathrm{c} 13, \mathrm{c} 14, \mathrm{c} 15)$.

${ }^{6} \mathrm{C} 18: 2$ other (C18:2 t,t NMID, t9,t12, t9,t13, t9,t12), where NMID = non-methylene-interrupted dienoic.

${ }^{7} \mathrm{ARA}=$ arachidonic acid.

in $\mathrm{B} 1$ raw milk; $6.50 \%$ in $\mathrm{B} 2)$. Linoleic acid (2.63\% vs. $3.82 \%)$ was higher $(P<0.05)$ in the $\mathrm{B} 2$ samples as was total CLA $(4.62 \mathrm{mg} / \mathrm{g}$ of fat vs. $7.19 \mathrm{mg} / \mathrm{g}$ of fat; Tables 5 and 6 ). From this total amount, $81.40 \%$ (Table 5) corresponded to $\mathrm{C} 18: 2$ c9t11 (RA) in B1 raw milk, whereas $84.31 \%$ corresponded to RA (Table 6$)$ in B2 $(P$ $>0.05)$. The second major CLA isomer was C18:2 t7c9, with $9.76 \%$ in $\mathrm{B} 1$ samples and $11.22 \%$ in $\mathrm{B} 2$ samples $(P<0.05)$.

Data in the present study are supported by other previous research works reporting that supplementation with different lipid sources is a useful way to increase the CLA content in milk (Loor et al., 2005; Nudda et al., 2006). The concentrations of RA and other CLA isomers are result of the biohydrogenation in the rumen of dietary PUFA and the action of the $\Delta^{9}$-desaturase on trans vaccenic acid in the mammary gland.

\section{Effect of Processing on the Fatty Acid Composition}

Both milk batches (B1 and B2) were processed to obtain pasteurized, UHT, STR, microwaved, and HP milk samples. The experiment was designed to test if milk fat could be affected by processing and if those changes may be associated with the fatty acid composition because preliminary results showed that B2 raw milk had higher concentration unsaturated FA than B1 raw milk.

Previous results from other authors are not conclusive. Herzallah et al. (2005a) conducted a study focused 
Table 3. trans monoenoic and C18:2 minor isomers composition (g/100 g of FA; means \pm SD) in B1 milk samples ${ }^{1}$

\begin{tabular}{|c|c|c|c|c|c|c|c|c|}
\hline Fatty acid ${ }^{2}$ & Raw & Past. & HTST & UHT1 & UHT2 & STR & HP & Micro. \\
\hline C18:1 t4 & $0.03 \pm 0.02$ & $0.03 \pm 0.01$ & $0.03 \pm 0.01$ & $0.03 \pm 0.01$ & $0.03 \pm 0.01$ & $0.03 \pm 0.01$ & $0.03 \pm 0.01$ & $0.03 \pm 0.01$ \\
\hline C18:1 t5 & $0.03 \pm 0.02$ & $0.03 \pm 0.02$ & $0.03 \pm 0.01$ & $0.03 \pm 0.02$ & $0.03 \pm 0.02$ & $0.03 \pm 0.02$ & $0.03 \pm 0.01$ & $0.03 \pm 0.01$ \\
\hline C18:1 t9 & $0.28 \pm 0.04$ & $0.28 \pm 0.07$ & $0.29 \pm 0.08$ & $0.24 \pm 0.03$ & $0.26 \pm 0.06$ & $0.34 \pm 0.07$ & $0.33 \pm 0.10$ & $0.32 \pm 0.06$ \\
\hline C18:1 t10 & $0.43 \pm 0.03$ & $0.45 \pm 0.06$ & $0.48 \pm 0.09$ & $0.43 \pm 0.04$ & $0.41 \pm 0.02$ & $0.49 \pm 0.07$ & $0.49 \pm 0.07$ & $0.45 \pm 0.08$ \\
\hline C18:1 t11 & $0.87 \pm 0.08$ & $0.85 \pm 0.04$ & $0.82 \pm 0.08$ & $0.78 \pm 0.06$ & $0.70 \pm 0.03$ & $0.84 \pm 0.06$ & $0.83 \pm 0.05$ & $0.80 \pm 0.09$ \\
\hline C18:1 t16 & $0.22 \pm 0.02$ & $0.24 \pm 0.03$ & $0.21 \pm 0.02$ & $0.23 \pm 0.02$ & $0.27 \pm 0.01$ & $0.22 \pm 0.02$ & $0.26 \pm 0.02$ & $0.23 \pm 0.03$ \\
\hline C18:1 c11 & $0.71 \pm 0.04$ & $0.67 \pm 0.08$ & $0.67 \pm 0.05$ & $0.66 \pm 0.04$ & $0.60 \pm 0.03$ & $0.71 \pm 0.06$ & $0.75 \pm 0.07$ & $0.66 \pm 0.06$ \\
\hline C18:1 c12 & $0.37 \pm 0.02$ & $0.33 \pm 0.05$ & $0.33 \pm 0.03$ & $0.32 \pm 0.03$ & $0.29 \pm 0.01$ & $0.35 \pm 0.03$ & $0.36 \pm 0.03$ & $0.31 \pm 0.04$ \\
\hline C18:1 c13 & $0.08 \pm 0.01$ & $0.07 \pm 0.01$ & $0.06 \pm 0.01$ & $0.07 \pm 0.01$ & $0.06 \pm 0.01$ & $0.08 \pm 0.01$ & $0.08 \pm 0.01$ & $0.07 \pm 0.01$ \\
\hline C18:1 c14 & $0.29 \pm 0.02$ & $0.28 \pm 0.04$ & $0.29 \pm 0.03$ & $0.27 \pm 0.03$ & $0.23 \pm 0.02$ & $0.31 \pm 0.03$ & $0.32 \pm 0.03$ & $0.28 \pm 0.03$ \\
\hline C18:1 c15 & $0.08 \pm 0.01$ & $0.08 \pm 0.01$ & $0.08 \pm 0.01$ & $0.08 \pm 0.01$ & $0.06 \pm 0.01$ & $0.09 \pm 0.01$ & $0.09 \pm 0.01$ & $0.08 \pm 0.01$ \\
\hline C18:2 t9c12 & $0.03 \pm 0.01$ & $0.05 \pm 0.02$ & $0.06 \pm 0.02$ & $0.05 \pm 0.02$ & $0.05 \pm 0.03$ & $0.03 \pm 0.01$ & $0.02 \pm 0.01$ & $0.03 \pm 0.02$ \\
\hline C18:2 c9c15 & $0.03 \pm 0.01$ & $0.02 \pm 0.01$ & $0.02 \pm 0.01$ & $0.02 \pm 0.01$ & $0.02 \pm 0.01$ & $0.02 \pm 0.01$ & $0.02 \pm 0.01$ & $0.02 \pm 0.01$ \\
\hline
\end{tabular}

${ }^{1}$ Past. $=$ low temperature pasteurization $\left(72^{\circ} \mathrm{C}, 30 \mathrm{~s}\right) ; \mathrm{HTST}=\mathrm{HTST}$ pasteurization $\left(85^{\circ} \mathrm{C}, 30 \mathrm{~s}\right) ; \mathrm{UHT} 1=\mathrm{UHT}$ processing $\left(135^{\circ} \mathrm{C}, 30 \mathrm{~s}\right) ; \mathrm{UHT} 2$ $=$ non-commercial $\mathrm{UHT}$ processing $\left(150^{\circ} \mathrm{C}, 5 \mathrm{~min}\right) ; \mathrm{STR}=$ sterilization $\left(121^{\circ} \mathrm{C}, 15 \mathrm{~min}\right) ; \mathrm{HP}=$ high pressure processing $\left(400 \mathrm{MPa}, 25^{\circ} \mathrm{C}, 15\right.$ $\min )$; Micro. $=$ microwave pasteurization $(650 \mathrm{~W}, 1.30 \mathrm{~min})$.

${ }^{2} \mathrm{t}=$ trans double bond; $\mathrm{c}=$ cis double bond.

${ }^{3} \mathrm{NMID}=$ non-methylene-interrupted dienoic.

Table 4. trans monoenoic and C18:2 minor isomers composition (g/100 g of FA; means $\pm \mathrm{SD}$ ) in B2 milk samples ${ }^{1}$

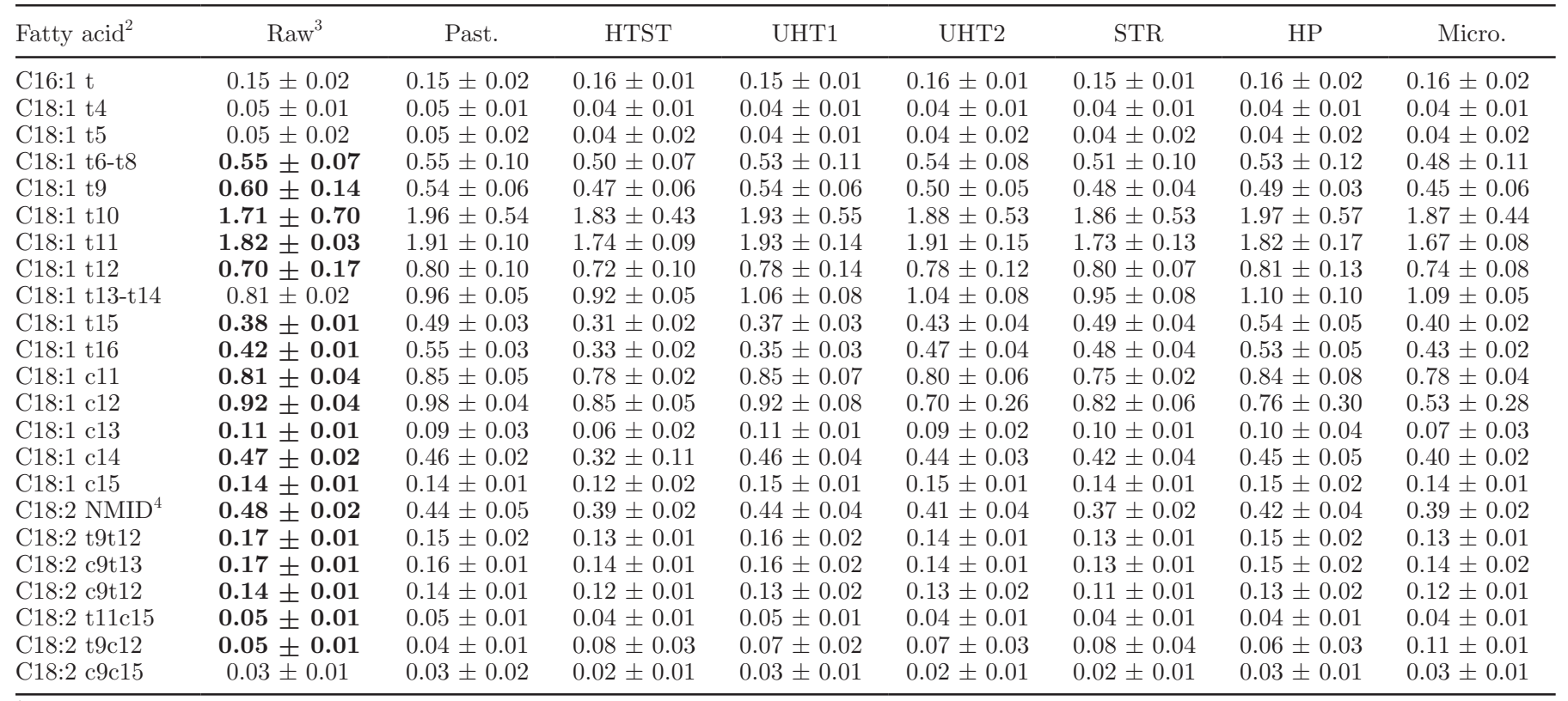

${ }^{1}$ Past. = low temperature pasteurization $\left(72^{\circ} \mathrm{C}, 30 \mathrm{~s}\right) ; \mathrm{HTST}=\mathrm{HTST}$ pasteurization $\left(85^{\circ} \mathrm{C}, 30 \mathrm{~s}\right)$; UHT1 $=\mathrm{UHT}$ processing $\left(135^{\circ} \mathrm{C}, 30 \mathrm{~s}\right) ; \mathrm{UHT} 2$ $=$ non-commercial $\mathrm{UHT}$ processing $\left(150^{\circ} \mathrm{C}, 5 \mathrm{~min}\right) ; \mathrm{STR}=$ sterilization $\left(121^{\circ} \mathrm{C}, 15 \mathrm{~min}\right) ; \mathrm{HP}=$ high pressure processing $\left(400 \mathrm{MPa}, 25^{\circ} \mathrm{C}, 15\right.$ $\min )$; Micro. = microwave pasteurization $(650 \mathrm{~W}, 1.30 \mathrm{~min})$.

${ }^{2} \mathrm{t}=$ trans double bond; $\mathrm{c}=$ cis double bond.

${ }^{3}$ Bold numbers in the raw milk sample column indicate significant differences among batches $(P<0.05)$.

${ }^{4} \mathrm{NMID}=$ non-methylene-interrupted dienoic. 
Table 5. Total CLA (mg/g of fat) and isomers distribution (g of isomer/100 g of CLA; means \pm SD) in B1 milk samples ${ }^{1}$

\begin{tabular}{|c|c|c|c|c|c|c|c|c|}
\hline Item $^{2}$ & Raw & Past. & HTST & UHT1 & UHT2 & STR & $\mathrm{HP}$ & Micro. \\
\hline $\begin{array}{l}\text { Total CLA } \\
\% \text { CLA isomers }\end{array}$ & $4.61 \pm 0.12^{\mathrm{ab}}$ & $4.51 \pm 0.20^{\mathrm{ab}}$ & $4.84 \pm 0.05^{\mathrm{b}}$ & $4.39 \pm 0.09^{\mathrm{a}}$ & $4.37 \pm 0.19^{\mathrm{a}}$ & $4.55 \pm 0.06^{\mathrm{a}}$ & $4.53 \pm 0.26^{\mathrm{a}}$ & $4.42 \pm 0.07^{\mathrm{ab}}$ \\
\hline t,t compounds & $7.64 \pm 1.18$ & $7.60 \pm 0.52$ & $8.18 \pm 0.44$ & $7.76 \pm 0.41$ & $8.17 \pm 0.60$ & $8.66 \pm 0.76$ & $7.66 \pm 0.29$ & $7.59 \pm 0.20$ \\
\hline 11,13 & $1.31 \pm 0.01$ & $1.17 \pm 0.12$ & $1.16 \pm$ & $1.15 \pm 0.15$ & $1.19 \pm 0.04$ & $1.18 \pm 0.10$ & $1.25 \pm 0.15$ & $1.13=$ \\
\hline 10,12 & $2.14 \pm 0.09$ & $2.08 \pm 0.09$ & $2.11 \pm$ & $1.99 \pm$ & $1.98 \pm$ & $2.12 \pm 0.12$ & $2.01 \pm 0.07$ & $1.96 \pm 0.07$ \\
\hline 9,11 & $1.43 \pm 0.34^{\mathrm{a}}$ & $1.55 \pm 0.21^{\mathrm{a}}$ & $1.77 \pm 0.10^{\mathrm{ab}}$ & $1.81 \pm 0.06^{\mathrm{ab}}$ & $1.95 \pm 0.29^{\mathrm{ab}}$ & $2.16 \pm 0.29^{\mathrm{b}}$ & $1.49 \pm 0.07^{\mathrm{a}}$ & $1.58 \pm 0.12^{\mathrm{a}}$ \\
\hline 12,14 & $0.39 \pm 0.02$ & $0.47 \pm 0.06$ & $0.46 \pm 0.07$ & $0.69 \pm 0.17$ & $0.71 \pm 0.20$ & $0.60 \pm 0.11$ & $0.55 \pm 0.10$ & $0.58 \pm 0.19$ \\
\hline 11,13 & $0.80 \pm 0.24$ & $0.62 \pm 0.13$ & $0.54 \pm 0.04$ & $0.74 \pm 0.20$ & $0.71 \pm 0.19$ & $0.72 \pm 0.11$ & $0.87 \pm 0.30$ & $0.76 \pm 0.24$ \\
\hline 9,11 & $81.40 \pm 0.94^{\mathrm{a}}$ & $81.49 \pm 0.26^{\mathrm{a}}$ & $81.04 \pm 0.36^{\mathrm{ab}}$ & $80.96 \pm 0.25^{\mathrm{ab}}$ & $80.62 \pm 0.51^{\mathrm{ab}}$ & $80.05 \pm 0.60^{\mathrm{b}}$ & $81.01 \pm 0.43^{\mathrm{ab}}$ & $81.20 \pm 0.52^{\mathrm{a}}$ \\
\hline 7,9 & $9.76 \pm 0.02$ & $9.82 \pm 0.61$ & $9.77 \pm 0.32$ & $9.85 \pm 0.34$ & $9.78 \pm 0.15$ & $9.99 \pm 0.27$ & $9.92 \pm 0.31$ & $9.88 \pm 0.37$ \\
\hline
\end{tabular}

${ }^{\mathrm{a}, \mathrm{b}}$ Values within a row with different superscripts differ significantly $(P<0.05)$.

${ }^{1}$ Past. $=$ low temperature pasteurization $\left(72^{\circ} \mathrm{C}, 30 \mathrm{~s}\right) ; \mathrm{HTST}=\mathrm{HTST}$ pasteurization $\left(85^{\circ} \mathrm{C}, 30 \mathrm{~s}\right) ; \mathrm{UHT} 1=\mathrm{UHT}$ processing $\left(135^{\circ} \mathrm{C}, 30 \mathrm{~s}\right) ; \mathrm{UHT} 2$ $=$ non-commercial $\mathrm{UHT}$ processing $\left(150^{\circ} \mathrm{C}, 5 \mathrm{~min}\right) ; \mathrm{STR}=$ sterilization $\left(121^{\circ} \mathrm{C}, 15 \mathrm{~min}\right) ; \mathrm{HP}=$ high pressure processing $\left(400 \mathrm{MPa}, 25^{\circ} \mathrm{C}, 15\right.$ $\min )$; Micro. $=$ microwave pasteurization $(650 \mathrm{~W}, 1.30 \mathrm{~min})$.

${ }^{2} \mathrm{t}, \mathrm{t}=$ trans trans; $\mathrm{ct} / \mathrm{tc}=$ cis trans/trans cis.

on pasteurized, UHT, boiled, and microwaved milk, reporting no changes in the concentrations of total SFA, MUFA, and PUFA. Those authors concluded that the continuous aqueous phase of milk acts as an oxygen barrier that hinders the oxidation reactions, and therefore triglycerides are not altered during processing. In further studies from other authors, cow milk naturally enriched in RA was assayed in the elaboration of UHT milk (Jones et al., 2005). The provided FA composition also showed absence of variations after processing. In a similar assay, Lynch et al. (2005) did not find alterations in pasteurized milk naturally enriched in C18:1 t11 and PUFA.
According to the results in the present research, the total concentration of SFA, MUFA, and PUFA (Tables 1 and 2) remained stable when milk from both batches were processed to obtain pasteurized, HTST, UHT1, UHT2, STR, HP, and microwave pasteurized milks in agreement with the previously cited studies. Furthermore, when the detailed FA composition was examined, stability was found for most of the compounds in all samples.

However, CLA concentration in both B1 and B2 milks (Tables 5 and 6 ) increased after HTST processing $(P<0.05)$. These variations represented an increment of $5 \%$ for B1 and $11 \%$ for B2 compared with the raw

Table 6. Total CLA (mg/g of fat) and isomers distribution ( $\mathrm{g}$ of isomer/100 $\mathrm{g}$ of CLA; means $\pm \mathrm{SD}$ ) in B2 milk samples ${ }^{1}$

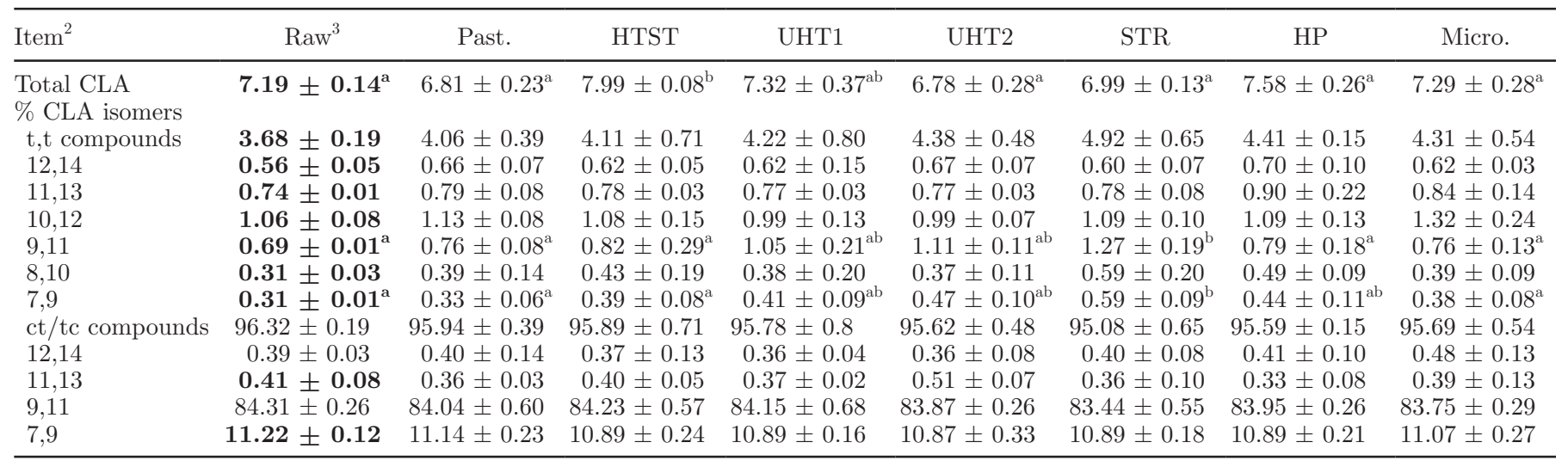

\footnotetext{
${ }^{\mathrm{a}, \mathrm{b}}$ Values within a row with different superscripts differ significantly $(P<0.05)$.
}

${ }^{1}$ Past. = low temperature pasteurization $\left(72^{\circ} \mathrm{C}, 30 \mathrm{~s}\right) ; \mathrm{HTST}=\mathrm{HTST}$ pasteurization $\left(85^{\circ} \mathrm{C}, 30 \mathrm{~s}\right) ; \mathrm{UHT} 1=\mathrm{UHT}$ processing $\left(135^{\circ} \mathrm{C}, 30 \mathrm{~s}\right) ; \mathrm{UHT} 2$ $=$ non-commercial $\mathrm{UHT}$ processing $\left(150^{\circ} \mathrm{C}, 5 \mathrm{~min}\right) ; \mathrm{STR}=$ sterilization $\left(121^{\circ} \mathrm{C}, 15 \mathrm{~min}\right) ; \mathrm{HP}=$ high pressure processing $\left(400 \mathrm{MPa}, 25^{\circ} \mathrm{C}, 15\right.$ $\min )$; Micro. $=$ microwave pasteurization $(650 \mathrm{~W}, 1.30 \mathrm{~min})$.

${ }^{2} \mathrm{t}, \mathrm{t}=$ trans trans; $\mathrm{ct} / \mathrm{tc}=$ cis trans/trans cis.

${ }^{3}$ Bold numbers in raw milk sample column indicate significant differences among batches $(P<0.05)$; superscript bold letters within a row for significant differences by processing $(P<0.05)$. 
milk samples. The contents of C18:2 c9t11 (Tables 1 and 2) showed a trend to increase in the HTST samples from $0.39 \%$ in raw milk from $\mathrm{B} 1$ to $0.43 \%$ and $0.84 \%$ in raw milk from $\mathrm{B} 2$ to $0.88 \%(0.05<P<0.1)$. The concentration of C18:2 c9c12 also showed lower levels after elaboration (2.63\% raw milk from B1 vs. $2.49 \%$ in HTST; $3.82 \%$ raw milk from B2 vs. $3.57 \%$; $0.05<$ $P<0.1)$.

A significant alteration of the distribution of CLA isomers in the STR samples was found: the proportion of C18:2 t9t11 (Table 5) changed from $1.43 \mathrm{~g}$ of isomer/100 g of CLA in B1 raw milk to $2.16 \mathrm{~g}$ of isomer/100 g of CLA, representing a variation of $51 \%$. This change was accompanied with a lower C18:2 c9t11 (RA) concentration (81.40 g of RA/100 g of CLA in B1 raw milk vs. $80.05 \mathrm{~g}$ of RA/100 g of CLA in STR samples). The level of this compound was also affected $(P<0.05)$ in B2 samples $(0.69 \mathrm{~g}$ of $\mathrm{C} 18: 2 \mathrm{t} 9 \mathrm{t} 11 / 100 \mathrm{~g}$ of CLA to $1.27 \mathrm{~g}$ of C18:2 t9t11/100 g of CLA; Table 6 ), whereas variation of RA was not significant (84.30 $\mathrm{g}$ of RA/100 $\mathrm{g}$ of CLA to $83.44 \mathrm{~g}$ of C18:2 RA/100 $\mathrm{g}$ of CLA; $P>0.05)$. The present results can be explained by taking into account that trans double bonds are more thermodynamically stable than cis. Thus, previous studies found that methyl linoleate can isomerize to C18:2 t9t11 and 18:2 t10t12 after heating at 200 and $220^{\circ} \mathrm{C}$ for $24 \mathrm{~h}$ (Destaillats and Angers, 2005). Assays with trilinolein suggested that reactions occur in the triglyceride molecule without previous release of the FA (Christy, 2010). In food such as milk butter, heating at $225^{\circ} \mathrm{C}$ for $15 \mathrm{~min}$ leads to isomerizations of $\mathrm{C} 18: 1$ c9 to $\mathrm{C} 18: 1 \mathrm{t} 9$, whereas $200^{\circ} \mathrm{C}$ for $2 \mathrm{~h}$ resulted in a sigmatropic rearrangement of rumenic and C18:2 t10, c12 into trans, trans CLA compounds (Precht et al., 1999; Destaillats et al., 2005). All these reactions were oxidative in nature. Because the observed changes were greater in the B2 samples than in B1, this seems to be related to the higher PUFA concentration in those samples. Thus, a high amount of substrate is available for the reaction.

Furthermore, in research works conducted with CLAfortified milks, Campbell et al. (2003) found that pasteurization diminished the concentration of RA whereas Rodriguez-Alcalá and Fontecha (2007) described that thermal processing of CLA enriched dairy products resulted in higher contents of trans, trans isomers, pointing to sigmatropic rearrangement reactions of the CLA fraction.

However, other authors found results different from those in the present research, regarding the total CLA content (Herzallah et al., 2005b): elaboration of microwave and UHT processing resulted in lower contents of total CLA when compared with untreated samples.
Low temperature treatments such as pasteurization create oxidative environments caused by oxygen solubility, which is inversely proportional to temperature, whereas $\mathrm{H}^{+}$proton donors proteins are denatured less than at higher temperatures (Giroux et al., 2008). According to this, the obtained results may suggest that in HTST processing, oxidative reactions leads to isomerization of linoleic acid into CLA isomers, while at sterilization temperatures, denatured proteins acting as $\mathrm{H}^{+}$proton donors are responsible for the sigmatropic rearrangement of the CLA fraction. It is stated that oxidation of thiol groups from proteins to produce thiyl radicals can lead to the formation of trans double bonds in FA (Geißler et al., 2003).

\section{CONCLUSIONS}

The results of the present study clearly showed a differential effect of processing on the CLA fraction of dairy fat. Although total CLA concentration increased in the elaboration of HTST milk, sterilization processing led to alterations in the distribution of these fatty acids, leading to higher concentrations of C18:2 t9t11 resulting from a possible sigmatropic rearrangement of RA. The PUFA concentration of the milk affects the extent of those changes.

\section{ACKNOWLEDGMENTS}

This work was supported by AGL-2011-26713 project.

\section{REFERENCES}

Alonso, L., J. Fontecha, L. Lozada, M. J. Fraga, and M. Juarez. 1999. Fatty acid composition of caprine milk: Major, branched-chain, and trans fatty acids. J. Dairy Sci. 82:878-884.

Bauman, D. E., I. H. Mather, R. J. Wall, and A. L. Lock. 2006. Major advances associated with the biosynthesis of milk. J. Dairy Sci. 89:1235-1243.

Calder, P. C. 2006. n-3 Polyunsaturated fatty acids, inflammation, and inflammatory diseases. Am. J. Clin. Nutr. 83:1505S-1519S.

Campbell, W., M. A. Drake, and D. K. Larick. 2003. The impact of fortification with conjugated linoleic acid (CLA) on the quality of fluid milk. J. Dairy Sci. 86:43-51.

Chawla, R., G. R. Patil, and A. K. Singh. 2011. High hydrostatic pressure technology in dairy processing: A review. J. Food Sci. Technol. 48:260-268.

Christy, A. A. 2010. Isomerization of 9c11t/10t12c CLA in triacylglycerols. Lipids 45:733-741.

Datta, N., M. G. Hayes, H. C. Deeth, and A. L. Kelly. 2005. Significance of frictional heating for effects of high pressure homogenisation on milk. J. Dairy Res. 72:393-399.

Destaillats, F., and P. Angers. 2005. Thermally induced formation of conjugated isomers of linoleic acid. Eur. J. Lipid Sci. Technol. 107:167-172.

Destaillats, F., C. Japiot, P. Y. Chouinard, J. Arul, and P. Angers. 2005. Rearrangement of rumenic acid in ruminant fats: A marker of thermal treatment. J. Dairy Sci. 88:1631-1635. 
Elgersma, A., S. Tamminga, and G. Ellen. 2006. Modifying milk composition through forage. Anim. Feed Sci. Technol. 131:207-225.

Geißler, C., O. Brede, and J. Reinhardt. 2003. cis-trans-Isomerization of unsaturated fatty acids during [gamma]-irradiation of barley grains. Radiat. Phys. Chem. 67:105-113.

Giroux, H. J., J. B. St-Amant, P. Fustier, J. M. Chapuzet, and M. Britten. 2008. Effect of electroreduction and heat treatments on oxidative degradation of a dairy beverage enriched with polyunsaturated fatty acids. Food Res. Int. 41:145-153.

Hagen, R. M., A. Rhodes, and M. R. Ladomery. 2013. Conjugated linoleate reduces prostate cancer viability whereas the effects of oleate and stearate are cell line-dependent. Anticancer Res. 33:4395-4400.

Harris, W. S. 2008. The omega-3 index as a risk factor for coronary heart disease. Am. J. Clin. Nutr. 87:1997S-2002S.

Haug, A., A. T. Hostmark, and O. M. Harstad. 2007. Bovine milk in human nutrition-A review. Lipids Health Dis. 6:25.

Herzallah, S. M., K. M. Al-Ismail, and M. A. Humeid. 2005a. Influence of some heating and processing methods on fatty acid profile of milk and other dairy products. J. Food Agric. Environ. 3:103-107.

Herzallah, S. M., M. A. Humeid, and K. M. Al-Ismail. 2005b. Effect of heating and processing methods of milk and dairy products on conjugated linoleic acid and trans fatty acid isomer content. J. Dairy Sci. 88:1301-1310.

Ip, C., S. P. Briggs, A. D. Haegele, H. J. Thompson, J. Storkson, and J. A. Scimeca. 1996. The efficacy of conjugated linoleic acid in mammary cancer prevention is independent of the level or type of fat in the diet. Carcinogenesis 17:1045-1050.

ISO-IDF. 2001. ISO 14156-IDF 172:2001: Milk and milk productsExtraction methods for lipids and liposoluble compounds. International Organization for Standardization (ISO), Geneva, Switzerland, and International Dairy Federation (IDF), Brussels, Belgium.

ISO-IDF. 2002. ISO 15884-IDF:182:2002: Milk fat-Preparation of fatty acid methyl esters. International Organization for Standardization (ISO), Geneva, Switzerland, and International Dairy Federation (IDF), Brussels, Belgium.

Jenkins, T. C., and M. A. McGuire. 2006. Major advances in nutrition: Impact on milk composition. J. Dairy Sci. 89:1302-1310.

Jones, E. L., K. J. Shingfield, C. Kohen, A. K. Jones, B. Lupoli, A. S. Grandison, D. E. Beever, C. M. Williams, P. C. Calder, and P. Yaqoob. 2005. Chemical, physical, and sensory properties of dairy products enriched with conjugated linoleic acid. J. Dairy Sci. 88:2923-2937.

Lawless, F., J. J. Murphy, D. Harrington, R. Devery, and C. Stanton. 1998. Elevation of conjugated cis-9,trans-11-octadecadienoic acid in bovine milk because of dietary supplementation. J. Dairy Sci. 81:3259-3267.

Li, B., Z. H. Wang, F. C. Li, and X. Y. Lin. 2007. Milk fat content was changed by ruminal infusion of mixed VFAs solutions with different acetate/propionate ratios in lactating goats. Small Rumin. Res. $72: 11-17$.

Loor, J. J., A. Ferlay, A. Ollier, M. Doreau, and Y. Chilliard. 2005. Relationship among trans and conjugated fatty acids and bovine milk fat yield due to dietary concentrate and linseed oil. J. Dairy Sci. 88:726-740.

Lynch, J. M., A. L. Lock, D. A. Dwyer, R. Noorbaksh, D. M. Barbano, and D. E. Bauman. 2005. Flavor and stability of pasteurized milk with elevated levels of conjugated linoleic acid and vaccenic acid. J. Dairy Sci. 88:489-498.
McNamee, B. F., A. M. Fearon, and J. Pearce. 2002. Effect of feeding oilseed supplements to dairy cows on ruminal and milk fatty acid composition. J. Sci. Food Agric. 82:677-684.

Moltó-Puigmartí, C., M. Permanyer, A. I. Castellote, and M. C. López-Sabater. 2011. Effects of pasteurisation and high-pressure processing on vitamin $\mathrm{C}$, tocopherols and fatty acids in mature human milk. Food Chem. 124:697-702.

Nishida, C., R. Uauy, S. Kumanyika, and P. Shetty. 2004. The Joint WHO/FAO Expert Consultation on diet, nutrition and the prevention of chronic diseases: Process, product and policy implications. Public Health Nutr. 7(1a):245-250.

Nudda, A., G. Battacone, M. G. Usai, S. Fancellu, and G. Pulina. 2006. Supplementation with extruded linseed cake affects concentrations of conjugated linoleic acid and vaccenic acid in goat milk. J. Dairy Sci. 89:277-282.

Pereda, J., V. Ferragut, J. M. Quevedo, B. Guamis, and A. J. Trujillo. 2007. Effects of ultra-high pressure homogenization on microbial and physicochemical shelf life of milk. J. Dairy Sci. 90:1081-1093.

Precht, D., J. Molkentin, and M. Vahlendieck. 1999. Influence of the heating temperature on the fat composition of milk fat with emphasis on cis-/trans-isomerization. Nahrung 43:25-33.

Rafalowski, R., Z. Zegarska, A. Kuncewicz, and Z. Borejszo. 2014. Oxidative stability of milk fat in respect to its chemical composition. Int. Dairy J. 36:82-87.

Rego, O. A., H. J. D. Rosa, P. V. Portugal, T. Franco, C. M. Vouzela, A. E. S. Borba, and R. J. B. Bessa. 2005. The effects of supplementation with sunflower and soybean oils on the fatty acid profile of milk fat from grazing dairy cows. Anim. Res. 54:17-24.

Rodríguez-Alcalá, L. M., T. Braga, F. X. Malcata, A. Gomes, and J. Fontecha. 2011. Quantitative and qualitative determination of CLA produced by bifidobacterium and lactic acid bacteria by combining spectrophotometric and $\mathrm{Ag}+-\mathrm{HPLC}$ techniques. Food Chem. 125:1373-1378.

Rodríguez-Alcalá, L. M., M. V. Calvo, and J. Fontecha. 2013a. A quick, optimized method for routine analysis of essential and trans-octadecenoic acids in edible fats and oils by GLC. J. Chromatogr. Sci. 51:70-81.

Rodríguez-Alcalá, L. M., and J. Fontecha. 2007. Hot topic: Fatty acid and conjugated linoleic acid (CLA) isomer composition of commercial CLA-fortified dairy products: Evaluation after processing and storage. J. Dairy Sci. 90:2083-2090.

Rodríguez-Alcalá, L. M., J. Fontecha, L. de la Hoz, V. S. N. da Silva, J. E. Carvalho, and M. T. B. Pacheco. 2013b. CLA-enriched milk powder reverses hypercholesterolemic risk factors in hamsters. Food Res. Int. 51:244-249.

Rodríguez-Alcalá, L. M., F. Harte, and J. Fontecha. 2009. Fatty acid profile and CLA isomers content of cow, ewe and goat milks processed by high pressure homogenization. Innov. Food Sci. Emerg. Technol. 10:32-36.

Urala, N., and L. Lahteenmaki. 2007. Consumers' changing attitudes towards functional foods. Food Qual. Prefer. 18:1-12.

Williams, C. M. 2000. Dietary fatty acids and human health. Anim. Res. 49:165-180.

Zamora, A., V. Ferragut, P. D. Jaramillo, B. Guamis, and A. J. Trujillo. 2007. Effects of ultra-high pressure homogenization on the cheese-making properties of milk. J. Dairy Sci. 90:13-23. 